\title{
Factors Associated With Residential Mobility in Children With Leukemia: Implications For Assigning Exposures
}

\author{
KEVIN Y. URAYAMA, MPH, JULIE VON BEHREN, MPH, PEGGY REYNOLDS, PHD, \\ ANDREW HERTZ, MONIQUE DOES, MPH, AND PATRICIA A. BUFFLER, PHD, MPH
}

\begin{abstract}
PURPOSE: In epidemiologic studies, neighborhood characteristics are often assigned to individuals based on a single residence despite the fact that people frequently move and, for most cancer outcomes, the relevant time-window of exposure is not known. The authors evaluated residential mobility patterns for a population-based series of childhood leukemia cases enrolled in the Northern California Childhood Leukemia Study.

METHODS: Complete residential history from 1 year before birth to date of diagnosis was obtained for 380 cases diagnosed between 1995 and 2002. All residences were assigned U.S. Census block group designations using a geographic information system.

RESULTS: Overall, two-thirds (65.8\%) of children had moved between birth and diagnosis, and onethird $(34.5 \%)$ moved during the first year of life. Approximately $25 \%$ of the mothers had moved during the year before the child's birth. Multivariable analysis indicated greater residential mobility to be associated with older age of the child at diagnosis, younger age of the mother at child's birth, and lower household income. Among those who had moved, residential urban/rural status for birth and diagnosis residences changed for about $20 \%$ of subjects, and neighborhood socioeconomic status for $35 \%$.

CONCLUSIONS: These results suggest that neighborhood attribute estimates in health studies should account for patterns of residential mobility. Estimates based on a single residential location at a single point in time may lead to different inferences.

Ann Epidemiol 2009;19:834-840. (C) 2009 Elsevier Inc. All rights reserved.
\end{abstract}

KEY WORDS: Childhood Leukemia, Epidemiology, Exposure Classification, Residential Mobility, Socioeconomic Status.

\section{INTRODUCTION}

It is becoming increasingly more common in epidemiological studies to use residential location to examine the effects of neighborhood characteristics and hazardous environmental exposures in relation to certain health outcomes, particularly with the expanding use of geographic information system tools (1). Studies examining the association between socioeconomic status (SES) and risk of childhood leukemia have used these methods to assign area-based measures of SES to children based on the geographic location of a single residence in time, usually birth, diagnosis, or death residence (2). Previous studies of childhood leukemia have also evaluated risks associated with proximity of a child's residence to sources of air pollution, traffic emissions (3), and agricultural pesticides (4). However, such data fail to account for the degree of misclassification that may

From the School of Public Health, University of California, Berkeley (K.Y.U. M.D., P.A.B); and Northern California Cancer Center, Berkeley (J.V.B., P.R., A.H.).

Address correspondence to: Kevin Y. Urayama, MPH, University of California, Berkeley, 2150 Shattuck Avenue, Suite 500, Berkeley, CA 94704. Tel.: 510-642-6398; Fax: 510-643-1735. E-mail: kurayama@ berkeley.edu.

Received November 6, 2008; accepted March 3, 2009. result from residential mobility of the subjects. Such concerns are compounded by the uncertainty regarding the potentially different effects of exposures during critical time windows of development, the importance of which is becoming more evident based on recent studies $(5,6)$.

This study takes advantage of an unusual opportunity to use complete residential histories from 1 year before birth to the date of diagnosis for a population-based series of childhood leukemia cases enrolled in the Northern California Childhood Leukemia Study (NCCLS). We evaluated both the degree of residential mobility and characteristics associated with mobility in this population. In addition, among children who moved, we evaluated the differences between the child's birth and diagnosis residence for two residential attributes of ongoing interest in the literature on childhood cancer: neighborhood levels of urbanization and SES.

\section{MATERIALS AND METHODS}

\section{Study Population}

The NCCLS is an ongoing case-control study designed to investigate the etiology of pediatric leukemias. Beginning in December of 1995, newly diagnosed childhood leukemia cases were rapidly ascertained from every major pediatric hospital 


\section{Selected Abbreviations and Acronyms}

$\mathrm{SES}=$ socioeconomic status

NCCLS $=$ Northern California Childhood Leukemia Study

GIS = geographic information system

$\mathrm{US}=$ United States

$\mathrm{CA}=$ California

MSA $=$ metropolitan statistical area

$\mathrm{RR}=$ rate ratios

$\mathrm{CI}=$ confidence interval

$\mathrm{CMSA}=$ Consolidated Metropolitan Statistical Area

located in a 17-county San Francisco Bay Area study region, which was expanded in 1999 to 35 counties in Northern and Central California. Initial comparisons with populationbased surveillance data from the California Cancer Registry (1997-1999) showed the NCCLS rapid case ascertainment protocol to have effectively identified nearly $90 \%$ of all ageeligible newly diagnosed childhood leukemia cases among residents of the study counties. Cases interviewed between 1995 and 2002 are included in this current analysis $(N=380)$. For each eligible case, the statewide birth registry maintained by the Center for Health Statistics of the California Department of Public Health was used to generate a list of randomly selected controls that matched the case on date of birth, sex, maternal race, and Hispanic status (has a biological parent who is Hispanic).

Cases and controls were considered eligible if they were under 15 years of age, resided in the study region at the date of diagnosis (or corresponding reference date for controls), had a parent or guardian who spoke either English or Spanish, and had no prior history of malignancy. Approximately $85 \%$ of eligible cases have consented to participate. Residential mobility patterns were presented for the case series only. The control group may be, by design, less mobile compared to the source population as a result of our control selection strategy that required tracing people from California birth addresses. A detailed description of control selection in the NCCLS is reported elsewhere (7).

The study protocol was approved by the Institutional Review Boards of University of California, Berkeley and all collaborating institutions, and a written informed consent was obtained for all participating subjects.

\section{Data Collection}

Interview data. A detailed in-home personal interview with the primary caretaker of each subject was conducted shortly after consent was obtained. Residential street addresses for all residences 1 month or longer were collected for children from birth to the date of diagnosis, and for mothers, 1 year before child's birth. Information pertaining to the child used to evaluate characteristics associated with residential mobility included age, sex, race/ethnicity, birth order, breast-feeding, daycare attendance, and complete residential history from birth to the time of diagnosis. Relevant parental characteristics included mother's age at child's birth, parental education, annual household income at the time of interview, mother's place of birth, mother's residences during the year before child's birth and whether she smoked some time during the 3 years after the child was born.

Residential attribute data. We geocoded all residences of the child from birth to the date of diagnosis. We used a geographic information system (GIS) to automatically match addresses to a road network and assigned a latitude/ longitude coordinate, which was then used to determine the corresponding U.S. Census block group. When possible, we manually located all addresses that could not automatically be matched. Geocoding was carried out using ArcView GIS software (8) and street databases (9-11). We did not attempt to geocode addresses outside California.

We used data from the U.S. Census Bureau to derive neighborhood measures of urbanization and SES. We assigned measures of urbanization to all residences (large metro, city, and rural/town) based on U.S. Census 1990 and 2000 block group data for residences with move-in dates during or before 1995 and move-in dates after 1995, respectively. The "large metro" category was defined as areas within a Metropolitan Statistical Area (MSA) or Consolidated Metropolitan Statistical Area (CMSA) of greater than or equal to one million people. The middle category, "city," included areas in MSA/CMSAs of less than one million people. The final category, "rural/town," included small towns $(<50,000$ people) and rural areas outside of census-designated urbanized areas.

We created a summary SES metric based on the 1990 and 2000 census data, incorporating block group measures of occupation, education and income $(9,12)$. To do this, we first ranked all California block groups separately by education level (percentage of adults over age 25 having completed a college degree or higher), income (median family income), and occupation (percentage of adults employed in managerial or professional occupations) according to quartiles based on the statewide adult population. This resulted in a score of one through four for each of these SES attributes (13). We then summed the scores for each attribute and categorized them into three groups based on quartiles: low SES (the lowest quartile of this score for the statewide population), medium SES (second and third quartiles), and high SES (the fourth and highest quartile).

\section{Statistical Analysis}

Subjects who reported having two or more residences between birth and diagnosis were considered movers. The Pearson $\chi^{2}$ test was used to compare the characteristics of movers and nonmovers among childhood leukemia cases. 
To evaluate factors associated with residential mobility, rate ratios (RR), and 95\% confidence intervals (CI) were calculated using multiple linear regression assuming a Poisson distribution for the number of child's residences from birth to diagnosis. A scaling factor was specified to adjust for the observed under-dispersion of the count data, a situation where the variance for the Poisson distribution of the data is smaller than the mean. Among childhood leukemia cases who moved, birth residence and diagnosis residence were compared in terms of two residential attributes: (1) urban/ rural status; and (2) SES. Kappa statistics were calculated to evaluate the categorical agreement of residential attribute levels between birth and diagnosis residence. The characteristics of children experiencing "no change" in residential attributes between birth and diagnosis residence were compared to children experiencing a change in residential attributes using the Pearson $\chi^{2}$ test.

\section{RESULTS}

Table 1 shows the patterns of residential moves among all childhood leukemia cases, and separately for younger and older age groups. Overall, 65.8\% (250/380) of the study children had two or more residences between birth and the date of diagnosis. As expected, child's residential mobility varied considerably by age with about $55 \%$ of children $0-4$ years of age having moved at least once compared to $80 \%$ of children 5-14 years of age. A large proportion of children that moved had at least one residence outside of the county of the birth residence (38.5\% among children 0-4 year of age and $46.6 \%$ among children 5-14 years of age), including areas outside of California and the United States. Among children who had moved, slightly greater than 50\% (131/250) moved for the first time during the first year of life. Finally, about $25 \%(101 / 380)$ of children's mothers moved during the year before the child's birth.

Table 2 presents the characteristics of movers (two or more residences between birth and diagnosis) and nonmovers (one residence). Compared to nonmovers, movers seemed to be older at diagnosis, have younger mothers, have parents with less formal education, have mothers who smoked, and have a diagnosis residence located in an area of lower SES. Also, there seemed to be a larger proportion of Hispanic and Black children than non-Hispanic White and "other" race/ethnicity among the movers. The results of the multivariable analyses presented in Table 3 indicate that, similarly, a greater number of residences is associated with older age of the child at diagnosis, younger age of the mother at child's birth, lower household income, and living in an area classified as "city" (vs. rural or town) at diagnosis. Analyses stratified by child's age at diagnosis produced similar results as the combined analysis (data not shown).

Among children who moved and had both birth and diagnosis residences geocoded $(N=201)$, we evaluated the differences between the child's birth and diagnosis residence for two attributes of interest, urban/rural status and SES. Tables 4 and 5 present the concordance and discordance

TABLE 1. Residential mobility and patterns of moves among all childhood leukemia cases stratified by child's age at diagnosis in the NCCLS (1995-2002)

\begin{tabular}{|c|c|c|c|c|c|c|}
\hline \multirow[b]{2}{*}{ Characteristic } & \multicolumn{2}{|c|}{ All Ages } & \multicolumn{2}{|c|}{ Ages 0-4 } & \multicolumn{2}{|c|}{ Ages 5-14 } \\
\hline & $N$ & $\%$ & $N$ & $\%$ & $N$ & $\%$ \\
\hline \multicolumn{7}{|l|}{ Mother moved during year before child's birth } \\
\hline No & 279 & 73.4 & 157 & 74.4 & 122 & 72.2 \\
\hline Yes & 101 & 26.6 & 54 & 25.6 & 47 & 27.8 \\
\hline \multicolumn{7}{|c|}{ Child's number of residences between birth and date of diagnosis } \\
\hline 1 (never moved) & 130 & 34.2 & 96 & 45.1 & 34 & 20.4 \\
\hline $2-3$ & 156 & 41.1 & 93 & 43.7 & 63 & 37.7 \\
\hline $4-6$ & 67 & 17.6 & 20 & 9.4 & 47 & 28.1 \\
\hline 7 or more & 27 & 7.1 & 4 & 1.9 & 23 & 13.8 \\
\hline$>1$ (has moved) & 250 & 65.8 & 117 & 54.9 & 133 & 79.6 \\
\hline Median number of residences (range) & \multicolumn{2}{|c|}{$2(1-14)$} & \multicolumn{2}{|c|}{$2(1-10)$} & \multicolumn{2}{|c|}{$3(1-14)$} \\
\hline \multicolumn{7}{|l|}{ Among children that have moved ${ }^{\dagger}$} \\
\hline Moved during the first year of life & 131 & 52.4 & 67 & 57.3 & 64 & 48.1 \\
\hline Moved out of US at least once & 26 & 10.4 & 9 & 7.7 & 17 & 12.8 \\
\hline Moved out of CA at least once $e^{\ddagger}$ & 49 & 19.6 & 16 & 13.7 & 33 & 24.8 \\
\hline Moved out of county of birth residence ${ }^{\S}$ & 107 & 42.8 & 45 & 38.5 & 62 & 46.6 \\
\hline Duration at birth residence (months $(\mathrm{SD}))^{*}$ & & & \multicolumn{2}{|c|}{$14.4(13.1)$} & \multicolumn{2}{|c|}{$22.7(24.3)$} \\
\hline Duration at diagnosis residence (months $(\mathrm{SD}))^{*}$ & & & \multicolumn{2}{|c|}{$14.7(12.4)$} & \multicolumn{2}{|c|}{$40.7(34.6)$} \\
\hline
\end{tabular}

$\mathrm{CA}=$ California; NCCLS $=$ Northern California Childhood Leukemia Study; SD = standard deviation; US = United States

* Mean number of months.

†Percent among children that have moved.

¥Includes subjects that have moved out of the US.

${ }^{8}$ Includes subjects that have moved out of the US and/or CA. 
TABLE 2. Characteristics of movers and non-movers in childhood leukemia cases enrolled in the NCCLS (1995-2002)

\begin{tabular}{lllll}
\hline \multirow{3}{*}{ Characteristics } & $\begin{array}{c}\text { Nonmovers } \\
(N=130)\end{array}$ & & $\begin{array}{c}\text { Movers } \\
(N=250)\end{array}$ \\
\cline { 2 - 2 } & $N \quad \%$ & & $\chi^{2} p^{*}$ \\
\hline
\end{tabular}

Child

Sex

$\begin{array}{llllll}\text { Male } & 72 & 55.4 & 133 & 46.8 & 0.685\end{array}$

Female

Race/ethnicity

$\begin{array}{llllll}\text { White, non-Hispanic } & 64 & 49.2 & 111 & 44.4 & 0.034\end{array}$

Hispanic

Black, non-Hispanic

Other

Age at diagnosis (yr)

$0-1$

2-4

5-9

10-14

Mean (SD)

Birth order

First

Second

Third or higher

Breast-feeding

No

Yes

Daycare attendance

No

Yes

Parent

Maternal age at child's birth (yr)

$<20$

20-24

25-29

30-34

$\geqslant 35$

Mean (SD) *

Maternal education

$\leqslant$ High school

Some college or other school

Bachelor's degree

Paternal education

$\leqslant$ High school

Some college or other school

Bachelor's degree

Household income $(\$ 1000 / \mathrm{yr})$

$<15$

$15-29$

$30-44$

$45-59$

$60-74$

$\geqslant 75$

Maternal place of birth

CA

US, not CA

Outside US

Mother moved during the year before child's birth

$\begin{array}{lllll}\text { No } & 97 & 74.6 & 182 & 73.1\end{array}$

Yes

$\begin{array}{llll}33 & 25.4 & 67 & 26.9\end{array}$
TABLE 2. (Continued)

\begin{tabular}{|c|c|c|c|c|c|}
\hline \multirow[b]{2}{*}{ Characteristics } & \multicolumn{2}{|c|}{$\begin{array}{l}\text { Nonmovers } \\
(N=130)\end{array}$} & \multicolumn{2}{|c|}{$\begin{array}{c}\text { Movers } \\
(N=250)\end{array}$} & \multirow[b]{2}{*}{$\chi^{2} p^{*}$} \\
\hline & $N$ & $\%$ & $N$ & $\%$ & \\
\hline \multicolumn{6}{|c|}{ Mother smoke postnatally ( $3 \mathrm{yr}$ ) } \\
\hline No & 114 & 87.7 & 196 & 78.7 & \multirow{2}{*}{0.035} \\
\hline Yes & 16 & 12.3 & 53 & 21.3 & \\
\hline \multicolumn{6}{|l|}{ Residential area } \\
\hline \multicolumn{6}{|c|}{ Urban/rural at diagnosis residence } \\
\hline Rural or town & 31 & 24.0 & 61 & 24.9 & \multirow[t]{3}{*}{0.953} \\
\hline City & 30 & 23.3 & 59 & 24.1 & \\
\hline Large metro & 68 & 52.7 & 125 & 51.0 & \\
\hline \multicolumn{6}{|c|}{ SES at diagnosis residence } \\
\hline Low & 18 & 14.0 & 58 & 23.7 & \multirow[t]{3}{*}{0.067} \\
\hline Medium & 65 & 50.4 & 117 & 47.8 & \\
\hline High & 46 & 35.6 & 70 & 28.6 & \\
\hline
\end{tabular}

CA = California; NCCLS = Northern California Childhood Leukemia Study; $\mathrm{SD}=$ standard deviation; SES = socioeconomic status; US = United States.

${ }^{*} p$ determined using two-sided Pearson's $\chi^{2}$ test.

0.568 between the two residences in terms of these residential attributes. On average, approximately $80 \%$ of children who moved experienced no change in urban/rural status between the two residences $(\kappa=0.672, p<0.001)$. Of those who did experience change, a larger number of children seemed to move from large metro areas to rural areas (14 subjects) compared to moves in the opposite direction (0 subjects). For SES, approximately $64 \%$ of children maintained the same residential SES between birth and diagnosis residence $(\kappa=0.428, \quad p<0.001)$, a slightly lower percentage compared to what was observed for urban/rural status. Of those who did change in residential SES, the direction seemed to be from lower to higher SES.

When examining the characteristics associated with change in residential attributes between birth and diagnosis residence, daycare attendance appeared to be less common among children who experienced change in urban/rural status compared to children with no change (data not shown). Also, children who experienced change were more likely to have mothers that moved during the year before the child's birth. No marked associations were observed for changes in SES between birth and diagnosis residence (data not shown).

\section{DISCUSSION}

This descriptive analysis of residential mobility for a population-based series of California childhood leukemia cases showed subjects to be highly mobile, with $65.8 \%$ of subjects having changed residences at least once (55\% among children $0-4$ years of age and $80 \%$ among children $5-14$ years of age) during the period between birth and the date of diagnosis. In a study conducted in Northern England, Alexander et al. (14) observed that $30.8 \%$ of children with leukemia 
TABLE 3. Multivariable analysis using Poisson regression to model child's number of residences as a function of select characteristics of childhood leukemia cases enrolled in the NCCLS (1995-2002)

\begin{tabular}{|c|c|c|}
\hline Characteristics & $\mathrm{RR}$ & $95 \% \mathrm{CI}^{*}$ \\
\hline \multicolumn{3}{|l|}{ Child } \\
\hline \multicolumn{3}{|l|}{ Age at diagnosis (yr) } \\
\hline $0-1$ & 1.00 & Reference \\
\hline $2-4$ & 1.47 & $1.19,1.84$ \\
\hline $5-9$ & 2.07 & $1.66,2.60$ \\
\hline $10-14$ & 2.61 & $2.06,3.31$ \\
\hline \multicolumn{3}{|l|}{ Race/ethnicity } \\
\hline White, non-Hispanic & 1.00 & Reference \\
\hline Hispanic & 0.93 & $0.80,1.07$ \\
\hline Black, non-Hispanic & 0.92 & $0.67,1.26$ \\
\hline Other & 1.03 & $0.82,1.29$ \\
\hline \multicolumn{3}{|l|}{ Parent } \\
\hline \multicolumn{3}{|l|}{ Maternal age at child's birth (yr) } \\
\hline$<20$ & 1.00 & Reference \\
\hline $20-24$ & 0.77 & $0.64,0.92$ \\
\hline $25-29$ & 0.66 & $0.54,0.80$ \\
\hline $30-34$ & 0.55 & $0.44,0.68$ \\
\hline$\geqslant 35$ & 0.45 & $0.34,0.60$ \\
\hline \multicolumn{3}{|l|}{ Paternal education } \\
\hline$\leqslant$ High school & 1.00 & Reference \\
\hline Some college or other school & 0.84 & $0.71,1.01$ \\
\hline Bachelor's degree & 1.00 & $0.83,1.20$ \\
\hline \multicolumn{3}{|l|}{ Household income $(\$ 1000 / \mathrm{yr})$} \\
\hline$<15$ & 1.00 & Reference \\
\hline $15-29$ & 0.90 & $0.75,1.08$ \\
\hline $30-44$ & 0.85 & $0.70,1.04$ \\
\hline $45-59$ & 0.66 & $0.54,0.82$ \\
\hline $60-74$ & 0.65 & $0.51,0.84$ \\
\hline$\geqslant 75$ & 0.72 & $0.57,0.92$ \\
\hline \multicolumn{3}{|l|}{$\begin{array}{l}\text { Mother moved during the year } \\
\text { before child's birth }\end{array}$} \\
\hline No & 1.00 & Reference \\
\hline Yes & 1.07 & $0.94,1.22$ \\
\hline \multicolumn{3}{|l|}{ Residential area } \\
\hline \multicolumn{3}{|l|}{ Urban/rural at diagnosis residence } \\
\hline Rural or town & 1.00 & Reference \\
\hline City & 1.26 & $1.07,1.48$ \\
\hline Large metro & 1.09 & $0.94,1.28$ \\
\hline
\end{tabular}

$\mathrm{CI}=$ confidence interval; NCCLS $=$ Northern California Childhood Leukemia Study; $\mathrm{RR}=$ rate ratio; $\mathrm{SD}=$ standard deviation; $\mathrm{SES}=$ socioeconomic status. $* \mathrm{RR}$ and $95 \% \mathrm{CI}$ were determined using multiple linear regression adjusting for all variables in the table and assuming a Poisson distribution for number of child's residences. A scaling factor was specified to adjust for the under-dispersion of the data. Variables included in the initial model included child's age at diagnosis, child's race/ ethnicity, maternal age at child's birth, maternal education, paternal education, household income at diagnosis, mother moved during the year before child's birth, maternal smoking after child's birth, residential urban/rural status at diagnosis residence and residential SES at diagnosis residence.

and non-Hodgkin's lymphoma had changed residence by the age of 2 . The California children in our study were more mobile, with $47.1 \%$ of children having changed residence by the age of 2 years. In addition, we observed a greater proportion of mothers moving during the year before child's birth (26.6\%) than in the Northern England study (16.5\%). Our observation for mother's residential mobility during the
TABLE 4. Changes in residential urban/rural between birth and diagnosis residence in childhood leukemia cases enrolled in the NCCLS (1995-2002)

\begin{tabular}{|c|c|c|c|c|c|c|c|c|}
\hline & \multicolumn{6}{|c|}{ Birth Residence } & \multirow{3}{*}{\multicolumn{2}{|c|}{ Total }} \\
\hline & \multicolumn{2}{|c|}{ Rural/Town } & \multicolumn{2}{|c|}{ City } & \multicolumn{2}{|c|}{ Large Metro } & & \\
\hline & $N$ & $\%$ & $N$ & $\%$ & $N$ & $\%$ & & \\
\hline \multicolumn{9}{|c|}{ Diagnosis residence } \\
\hline Rural/town & 26 & 86.7 & 8 & 16.0 & 14 & 11.6 & 48 & 23.9 \\
\hline City & 4 & 13.3 & 37 & 74.0 & 8 & 6.6 & 49 & 24.4 \\
\hline Large metro & 0 & 0.0 & 5 & 10.0 & 99 & 81.8 & 104 & 51.7 \\
\hline Total & 30 & 14.9 & 50 & 24.9 & 121 & 60.2 & 201 & 100.0 \\
\hline
\end{tabular}

NCCLS $=$ Northern California Childhood Leukemia Study.

year before child's birth is more consistent with U.S. studies of birth defects that have reported findings ranging from 20\%-33\% (15-17). The authors of the Northern England study acknowledge, however, that their study population is likely to be less mobile compared to the general population because selection into the study required children to have been living in the same area at birth and at diagnosis. As expected, our results are similar to those from a study of leukemia in children less than 5 years of age conducted in San Diego, California that found that more than $50 \%$ of those young children had moved at least once between birth and diagnosis (18).

In this study, several factors were found to be associated with residential mobility including older age of the child, younger age of the mother at child's birth, lower household income, and diagnosis residence located in "city" compared to "rural or town." Somewhat unexpectedly, there did not seem to be a significant difference in the number of residences between the race/ethnicity groups after accounting for the influence of SES in the multivariable analysis. We are not aware of other studies in the epidemiologic literature that describe characteristics associated with child's residential mobility. However, studies evaluating the mobility of mothers during pregnancy have reported similar findings associated with age, race, and SES $(15,19)$. Urban/rural

TABLE 5. Changes in socioeconomic status between birth and diagnosis residence in childhood leukemia cases enrolled in the NCCLS (1995-2002)

\begin{tabular}{|c|c|c|c|c|c|c|c|c|}
\hline & \multicolumn{6}{|c|}{ Birth Residence } & & \\
\hline & \multicolumn{2}{|c|}{ Low } & \multicolumn{2}{|c|}{ Medium } & \multicolumn{2}{|c|}{ High } & & \\
\hline & $N$ & $\%$ & $N$ & $\%$ & $N$ & $\%$ & \multicolumn{2}{|c|}{ Total } \\
\hline \multicolumn{9}{|c|}{ Diagnosis residence } \\
\hline Low SES & 31 & 62.0 & 18 & 16.7 & 1 & 2.3 & 50 & 24.9 \\
\hline Medium SES & 16 & 32.0 & 67 & 62.0 & 11 & 25.6 & 94 & 46.8 \\
\hline High SES & 3 & 6.0 & 23 & 21.3 & 31 & 72.1 & 57 & 28.3 \\
\hline Total & 50 & 24.9 & 108 & 53.7 & 43 & 21.4 & 201 & 100.0 \\
\hline
\end{tabular}


status was not found to be associated with mobility in these studies of pregnant women.

Many studies of SES and childhood leukemia have used neighborhood measures of SES based on a single residence (2). A recent case-control study from Denmark, which was able to compare the risk of childhood leukemia associated with SES based on birth residence and diagnosis address, observed an increased leukemia risk for children born in low-income areas, but no difference based on diagnosis address or based on individual SES information (job class of parents) (20). This study highlights the fact that different inferences may be drawn by relying on a single residence at one point in time, and this may account for some of the inconsistencies in the literature on SES and childhood leukemia $(2,21)$.

Several studies, including one recent California statewide study, have shown that low-income neighborhoods and communities of color are more likely to be located near sources of pollution (22-24). This suggests that the changes in SES between birth and diagnosis residence observed in our study population may also be accompanied by changes in exposures to environmental pollutants, including motor vehicle emissions and industrial pollutants. Most previous studies have estimated such exposures based on either child's birth residence, residence at diagnosis, or current residence primarily due to the lack of residential history information. Our limited knowledge of the causes of childhood leukemia and the role of timing of exposure makes it difficult to assess which of these estimates may be most appropriate, and this resulting "misclassification" may contribute to the inconsistent results for many of these factors. In our comparison of child's birth and diagnosis residence with regard to two residential attributes, we found that a large proportion of subjects tended to move to areas of similar urban/rural status (80\%) and SES (64\%). However, this may be an overestimate because this analysis was limited to subjects with both birth and diagnosis residences geocoded, and as a result, excluded a small number of subjects born outside of California (2.9\%) and those with residences that could not be geocoded for some reason ( $\sim 10 \%$ of subjects' birth or diagnosis residences). Furthermore, this analysis does not evaluate changes in residential attributes for residences other than those at birth and diagnosis.

Our focus on a population-based series of childhood leukemia cases enrolled in the NCCLS as opposed to the controls was guided by our concern that the control group may be artificially less mobile compared to the case source population as a result of our control selection strategy. In preliminary analyses, cases do seem to be more mobile than controls, however, the degree to which this difference in mobility can be attributed to our selection strategy has not been determined. Although the two groups differed with respect to total number of residences, the factors associated with residential mobility among controls were largely similar to those presented here for cases. In addition, the concordance and discordance between birth and diagnosis residence with regard to urban/rural status and neighborhood SES did not seem to significantly differ from that of cases.

In summary, results of this descriptive analysis show this population-based series of cases to be highly residentially mobile, with patterns that differ by demographic subgroups. These results suggest that for epidemiologic studies of children, even very young ones, there are serious implications in using a single residential location to determine area-based exposure assignments. Additionally, in view of the uncertainties about which time periods may be of primary importance for leukemia risk and to examine exposures during different time periods, attempts should be made to account for patterns of residential mobility. With full residential histories of the children, area-based measures can be estimated for all residences, and changes in estimates due to residential mobility can be accounted for in the analysis. In the absence of pertinent residential history data in studies where children are suspected to be residentially mobile, one strategy may be to limit the study population to children with certain demographic characteristics that have been associated with less mobility, such as those identified in this analysis.

This study was supported by grants from the National Institute of Environmental Health Sciences (P42 ES04705, R01 ES09137) and the National Cancer Institute (R01 CA92674). We thank the participating hospitals and clinical collaborators. We would also like to acknowledge the participants of this study and the Northern California Childhood Leukemia Study staff for their effort and dedication.

\section{REFERENCES}

1. Boscoe FP, Ward MH, Reynolds P. Current practices in spatial analysis of cancer data: data characteristics and data sources for geographic studies of cancer. Int J Health Geogr. 2004;3:28.

2. Poole C, Greenland S, Luetters C, Kelsey JL, Mezei G. Socioeconomic status and childhood leukemia: a review. Int J Epidemiol. 2006;35:370-384.

3. Raaschou-Nielsen O, Reynolds P. Air pollution and childhood cancer: a review of the epidemiological literature. Int J Cancer. 2006;118: 2920-2929.

4. Alavanja MC, Ward MH, Reynolds P. Carcinogenicity of agricultural pesticides in adults and children. J Agromedicine. 2007;12:39-56.

5. Buffler PA, Kwan ML, Reynolds P, Urayama KY. Environmental and genetic risk factors for childhood leukemia: appraising the evidence. Cancer Invest. 2005;23:60-75.

6. McNally RJ, Parker L. Environmental factors and childhood acute leukemias and lymphomas. Leuk Lymphoma. 2006;47:583-598.

7. Ma X, Buffler PA, Layefsky M, Does MB, Reynolds P. Control selection strategies in case-control studies of childhood diseases. Am J Epidemiol. 2004;159:915-921.

8. Environmental Systems Research Institute I. ArcView, version 3.3. Redlands (CA): ESRI; 2000.

9. United States Bureau of the Census. TIGER2000 [data file]. Washington DC: US Census Bureau; 2002. 
10. Geographic Data Technologies GD. GDT Home Page: Available at: http://www.geographic.com/home/index.cfm.

11. Navigational Technologies. NavTech Home Page. Available at: http:// www.navtech.com.

12. United States Bureau of the Census. Census of population and housing. Modified age/race, sex and Hispanic origin (MARS) state and county file [data file] 1990. Washington, DC: US Bureau of the Census; 1992.

13. Reynolds P, Hurley SE, Quach AT, Rosen H, Von Behren J, Hertz A, et al. Regional variations in breast cancer incidence among California women, 1988-1997. Cancer Causes Control. 2005;16:139-150.

14. Alexander FE, McKinney PM, Cartwright RA. Migration patterns of children with leukemia and non-Hodgkin's lymphoma in three areas of northern England. J Public Health Med. 1993;15:9-15.

15. Canfield MA, Ramadhani TA, Langlois PH, Waller DK. Residential mobility patterns and exposure misclassification in epidemiologic studies of birth defects. J Expo Sci Environ Epidemiol. 2006;16:538-543.

16. Khoury MJ, Stewart W, Weinstein A, Panny S, Lindsay P, Eisenberg M. Residential mobility during pregnancy: implications for environmental teratogenesis. J Clin Epidemiol. 1988;41:15-20.

17. Shaw GM, Malcoe LH. Residential mobility during pregnancy for mothers of infants with or without congenital cardiac anomalies: a reprint. Arch Environ Health. 1992;47:236-238.
18. Reynolds P, Elkin E, Scalf R, Von Behren J, Neutra RR. A case-control pilot study of traffic exposures and early childhood leukemia using a geographic information system. Bioelectromagnetics(Suppl 5). 2001:S58-68.

19. Fell DB, Dodds L, King WD. Residential mobility during pregnancy. Paediatr Perinat Epidemiol. 2004;18:408-414.

20. Raaschou-Nielsen O, Obel J, Dalton S, Tjonneland A, Hansen J. Socioeconomic status and risk of childhood leukemia in Denmark. Scand J Public Health. 2004;32:279-286.

21. Smith A, Roman E, Simpson J, Ansell P, Fear NT, Eden T. Childhood leukemia and socioeconomic status: fact or artefact? A report from the United Kingdom childhood cancer study (UKCCS). Int J Epidemiol. 2006;35:1504-1513.

22. Gunier RB, Hertz A, Von Behren J, Reynolds P. Traffic density in California: socioeconomic and ethnic differences among potentially exposed children. J Expo Anal Environ Epidemiol. 2003;13:240-246.

23. Neumann CM, Forman DL, Rothlein JE. Hazard screening of chemical releases and environmental equity analysis of populations proximate to toxic release inventory facilities in Oregon. Environ Health Perspect. 1998;106:217-226.

24. Perlin SA, Sexton K, Wong DW. An examination of race and poverty for populations living near industrial sources of air pollution. J Expo Anal Environ Epidemiol. 1999;9:29-48. 